\title{
Study on the Stress Relaxation Property of Degradable Polymer PLGA
}

\author{
Yang $\mathrm{Gao}^{1, \mathrm{a}}$, Yaguang $\mathrm{Huo}^{2, \mathrm{~b}}$ \\ ${ }^{1}$ School of Mechanical Engineering, Xi'an University of Science and Technology, Xi'an,710054, \\ China; \\ ${ }^{2}$ Modern Engineering Training Center, Chang'an University, Xi'an, 710064, China. \\ agaoyang_mec@163.com, bhuoyg01@163.com
}

Keywords: Degradable polymer, Stress relaxation, Hot embossing, Microstructure.

\begin{abstract}
Multi-Microstructures are designed as the carriers of controlled drug delivery system, and the degradable polymer is the preferred material. When the deform method is hot embossing, the viscoelasticity of degradable polymer becomes one of the important parameters which affect the quality of microstructure. So the viscoelasticity and stress relaxation property of polymer is researched in this paper by experiment, and the numerical material model was established based on the generalized Maxwell model with 5 units by the Finite Element Method, The simulation results show that the model is reasonable and give the base for the research of optimum forming parameters of microstructure forming by hot embossing.
\end{abstract}

\section{Introduction}

Biomaterials have played an enormous role in the success of medical devices, especially in the development of controlled drug delivery systems (CDDS). Polylactic acid-polyglycolic acid (PLGA) is one of the important degradable materials used in CDDS, which is the copolymer of polylactic acid (PLA) and polyglycolic acid (PGA). The formation of the PLGA microstructure is important because it is not only taking shape the drug loading cavities but also the part for bonding which makes the integrality of CDDS. The normally used method of PLGA formation is hot embossing.

Hot embossing is a widely used method for the fabrication of polymeric microstructures both in academia and in industry. In hot embossing process, the polymer does not need to be transported a long way into the molding tool, this reduces the shrinkage during cooling down and the friction forces acting on the micro structure during demolding. Thus more delicate micro structures with higher aspect ratios can be fabricated by the hot embossing. So the hot embossing was chose to form the microstructure for drug loading in this paper.

Since the polymer usually has macromolecular chains, so it is possesses stress relaxation and viscoelasticity in hot embossing process. Many researchers did efforts about the polymer formation by hot embossing. Heyderman et al. [1] studied the flow behavior of PMMA in the filling process of concave model; the pressure distribution of polymer materials in hot embossing was simulated based on 3 units viscoelastic model by Lin et al.[2]; According to the 24units generalized Maxwell viscoelastic model, the deformation of PMMA was researched with changing the pressure, temperature and time in hot embossing process by Hirai [3]; the effect of model structure and the material initial thickness on deformation quality were studied by Kim[4]; Lan [5] got the relaxation features of PC polymer at the glass transition temperature. Whereas, the above experimental and simulation researches about polymer formation are mostly focus on the materials of PMMA or PC, which glass transition temperature is higher relatively. The polymer of our research is PLGA, which glass transition temperature is $45-50^{\circ} \mathrm{C}$, and the deformation properties is time-related because the specific entropy elasticity of polymer chains. But the research on the viscoelasticity of PLGA is less, for the wildly use of PLGA in CDDS, it is necessary to give the investigation about the viscoelasticity of PLGA by hot embossing format. 
The aim of this paper is to research the relaxation property of PLGA by experimental research, and then, the generalized Maxwell viscoelastic model was established by the finite element method (FEM) to simulate the process of PLGA hot embossing.

\section{Stress Relaxation Experiment}

The PLGA material (monomer ratio PLA/PGA is 75/25) used in the stress relaxation experiments is purchased from LACTEL $\AA$ USA, which glass transition temperature is between $40-50^{\circ} \mathrm{C}$. PLGA test samples of $30 \times 5 \times 1.5 \mathrm{~mm}$ were fabricated in advance. Dynamic mechanical analyzer

(DMA-Q800) was used to measure the changes of relaxation modulus. In experiments, the constant stretch strain is $1 \%$ in length of the test sample, the relaxation time is $360 \mathrm{~s}$, the temperature range is $60-80{ }^{\circ} \mathrm{C}$ and the temperature interval is $5^{\circ} \mathrm{C}$. The experimental results about the PLGA normalized relaxation modulus in different temperatures are shown in Fig.1, where the $\mathrm{X}$ axis is the relaxation time and the $\mathrm{Y}$ axis is the normalized relaxation modulus which a normalization process was performed by dividing the measured stress in function of time $G(t)$ of the initial stress $G_{0}$.

75/25 PLGA

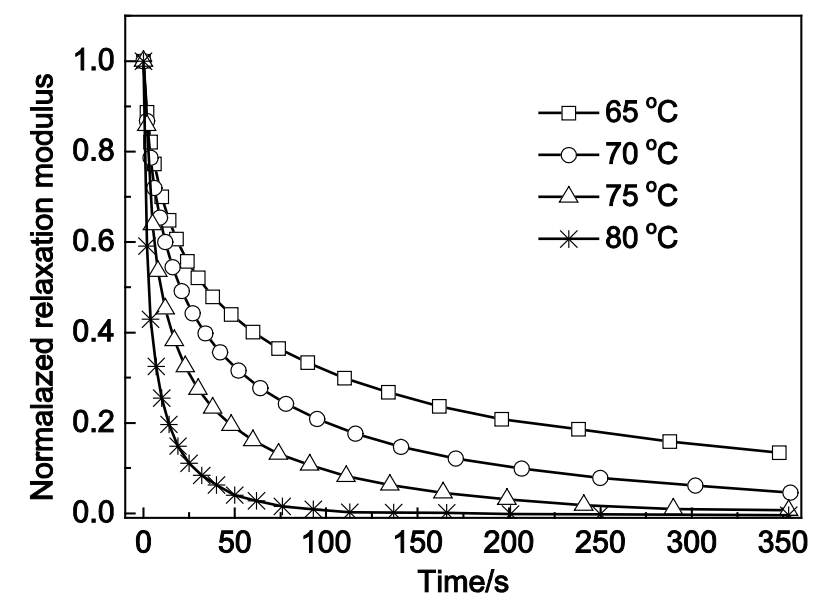

Fig. 1 Normalized relaxation modulus curves of PLGA (75/25) with different temperatures

As shown in Fig.1, the relaxation modulus reduced significantly as the time elapsed at every tested temperature. The decrease rate of the relaxation modulus is rapid in the early stage and slows down with the time goes. In another hand, the decrease rate of the relaxation modulus increased as the tested temperature increased, modulus decreased rapidly to a lower value and then unchanged at $80^{\circ} \mathrm{C}$. This is because the higher temperature increased the energy of polymer molecular movement, and the volume of polymer expansion occurs increasing the free space between molecules. Both effects are speed up the process of relaxation, thus shorten the relaxation time.

\section{The Master Curve of PLGA Stress Relaxation}

According to the time-temperature equivalence characteristics of polymer materials, the relaxation behaviors are equivalent with the relatively short period of time at higher temperature and long period of time at lower temperature. Thus, if one reference temperature was selected, the relaxation curves at other temperatures can shift overlap. The overlapped relaxation curve is the master curve which is the complete relaxation process for the polymer. For the amorphous polymer PLGA, the remove factor can be represented by the WLF equation [6]:

$$
\log _{10} a_{T}=\frac{-C_{1} \times\left(T-T_{0}\right)}{C_{2}+\left(T-T_{0}\right)}
$$

Where $a_{T}$ is the remove factor, $T_{0}$ is the reference temperature and $T$ is measuring temperatures in relaxation experiment except the reference temperature, $C_{1}$ and $C_{2}$ are the coefficients. 
So, the master relaxation modulus curve of $75 / 25$ PLGA at $65^{\circ} \mathrm{C}$ can be obtained by moving the other relaxation curves at different temperatures in logarithmic coordinates based on Fig. 1, which is shown by square-line in Fig. 2. Meanwhile, the coefficients $C_{1}$ and $C_{2}$ can be got by substituting the remove factor to Eq. (1): $C_{1}$ is 8.2 and $C_{2}$ is 85.6.

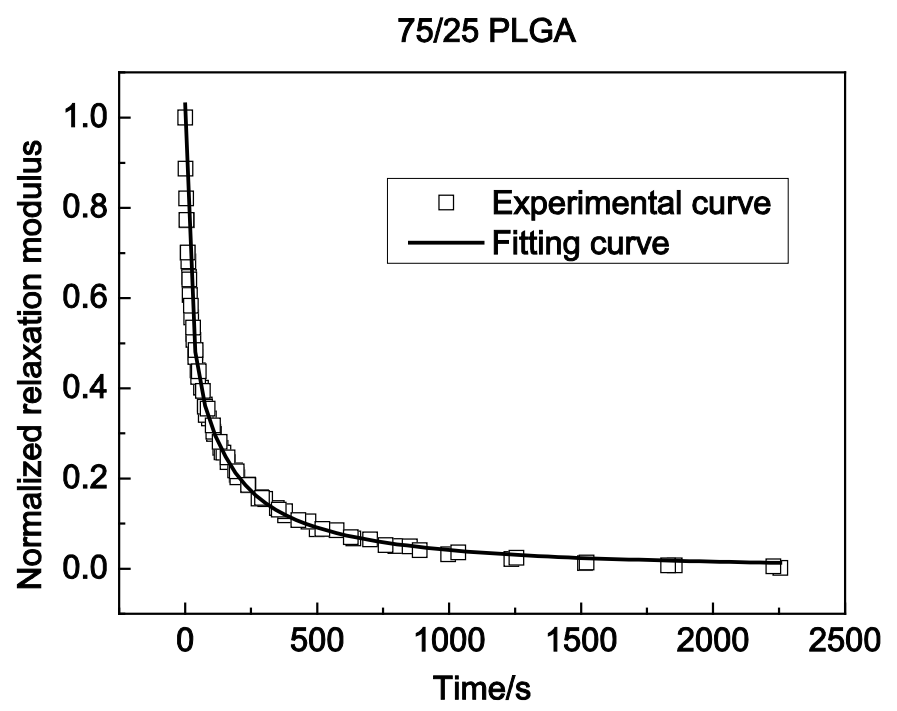

Fig. 2 The master relaxation curve of PLGA and its fitting curve at $65^{\circ} \mathrm{C}$

\section{The Viscoelastic Model of PLGA}

The finite element system ANSYS was used and the generalized Maxwell model was chose to model and simulate the formation behavior of PLGA substrate to microstructure in hot embossing process. The relaxation modulus of the generalized Maxwell model is shown as:

$$
G(t)=G_{\infty}+\sum_{i=1}^{n} G_{i} \exp \left(-\frac{t}{\tau_{i}}\right)
$$

Where $G(t)$ is the relaxation modulus, $G_{\infty}$ is the ultimate relaxation modulus, $G_{i}$ is the coefficient of relaxation modulus, $\tau_{i}$ is the relaxation time, and $n$ is the number of Maxwell units.

The non-linear fitting was conducted to the master curve of relaxation modulus, and the result is shown by smooth-line in Fig. 2. The correlation coefficient of the fitting curve and the experimental curve is 0.9987, which indicates that the generalized Maxwell model can simulate the relaxation behavior of PLGA material accurately.

The parameters of generalized Maxwell model with 5 units based on fitting curve are listed in the Tab. 1, which provided the basis to the subsequent simulated research about the forming process of PLGA.

Tab. 1 The parameters of generalized Maxwell model

\begin{tabular}{cccc}
\hline $\begin{array}{c}\text { Parameter } \\
S\end{array}$ & Fitting value & $\begin{array}{l}\text { Parameter } \\
\text { s }\end{array}$ & Fitting value \\
\hline$G_{\infty}$ & 0.00017 & -- & -- \\
$G_{1}$ & 0.16845 & $\tau_{1}$ & 1.21 \\
$G_{2}$ & 0.3181 & $\tau_{2}$ & 19.73 \\
$G_{3}$ & 0.31483 & $\tau_{3}$ & 113.27 \\
$G_{4}$ & 0.17621 & $\tau_{4}$ & 396.38 \\
$G_{5}$ & 0.05149 & $\tau_{5}$ & 1565.40 \\
\hline
\end{tabular}

For the symmetry shape of micro structure, half of the groove template was taken as the analysis object. The deepness of template, $\mathrm{H}$, is $540 \mu \mathrm{m}$; the half of width, $\mathrm{L} / 2$, is $60 \mu \mathrm{m}$ and the half of spacing, $\mathrm{S} / 2$, is $800 \mu \mathrm{m}$. In the analysis, supposed that there is no relative slip in the contact surface of the template and PLGA substrate. Boundary conditions at the left and the right boundary are symmetrical, 
the bottom of PLGA membrane was limited, and the constant pressure was applied on the template surface perpendicularly. The temperature distribution in this model is evenly. The material of template is PDMS, which elastic modulus is $3 \mathrm{MPa}$ and the Poisson's ratio is 0.499 . Fig. 3 shows the same template groove was filled by PLGA at 80 ond under the acting pressure $40 \mathrm{~N}$, the action time of pressure is 20s, 50s and 70s. As shown in Fig.3, there is a tendency that longer action time makes better quality of the microstructure. The microstructure model is $100 \%$ filled at 70 s.

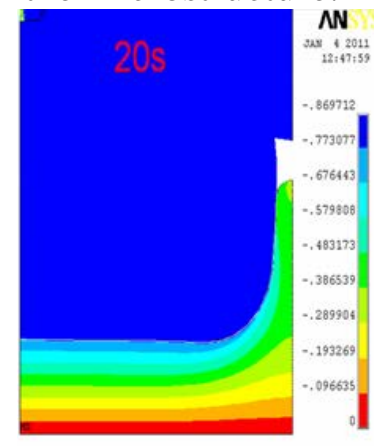

Fig. 3 The filling situation of PLGA at 80
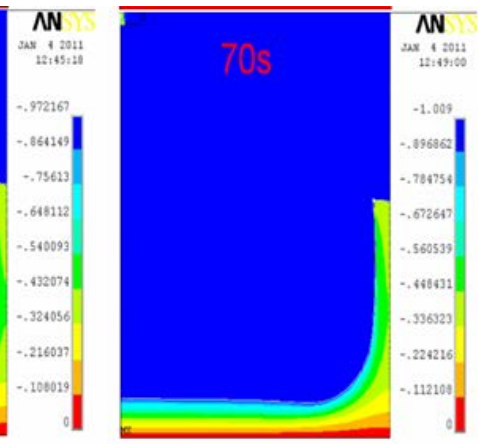

Q 2 ith different acting pressure

\section{Conclusions}

In this paper, the stress relaxation property of PLGA was studied. The relaxation modulus of 75/25 PLGA with the increasing temperatures was tested, and based on the WLF function, the master curve of stress relaxation was got with the reference temperature $65^{\circ} \mathrm{C}$ and the constants of $\mathrm{C}_{1}$ and $\mathrm{C}_{2}$ in WLF function were estimated: $C_{1}=8.2$ and $C_{2}=85.6$. For the viscoelasticity of PLGA, the hot embossing process was simulated using FEM based on the generalized Maxwell mold with 5 units. The above researches give the bases for the parameters optimization of PLGA microstructure formation by hot embossing.

\section{Acknowledgments}

The authors gratefully acknowledge the support of China Postdoctoral Science Foundation (2015M572579), Scientific Research Program Funded by Shaanxi Provincial Education Department (Program No. 2013JK1031), Cultivation Program (201224) and Doctoral Research and Start-up Program (2012QDJ026) Funded by Xi'an University of Science and Technology.

\section{References}

[1] Heyderman LJ, Schift H, David C, et al. Flow behaviour of thin polymer films used for hot embossing lithography. Microelectronic Engineering. 54 (2000) 229-245.

[2] Lin CR, Hung C. The characterisation and finite-element analysis of a polymer under hot pressing. International Journal of Advanced Manufacturing Technology. 20 (2002) 230-235.

[3] Hirai Y, Onishi Y, Tanabe T, et al. Pressure and resist thickness dependency of resist time evolutions profiles in nanoimprint lithography. Microelectronic Engineering. 85 (2008) 842-845.

[4] Kim NW, Kim KW, Sin HC. Finite element analysis of low temperature thermal nanoimprint lithography using a viscoelastic model. Microelectronic Engineering. 85 (2008) 1858-1865.

[5] Lan S, Lee HJ, Lee SH, et al. Experimental and numerical study on the viscoelastic property of polycarbonate near glass transition temperature for micro thermal imprint process. Materials and Design. 30 (2009) 3879-3884.

[6] Patel M, Soames M, Skinner AR, et al., Stress relaxation and thermogravimetric studies on room temperature vulcanised polysiloxane rubbers, Polymer Degradation and Stability, 83 (2004) 111-116. 